\title{
Molecular Dynamics Simulations of Electron-Alkali Cation Pairs in Bulk Water
}

\author{
François-Xavier Coudert, Pierre Archirel, and Anne Boutin* \\ Laboratoire de Chimie Physique UMR 8000, CNRS, Université de Paris-Sud 11, 91405 Orsay Cedex, France
}

Received: August 3, 2005; In Final Form: October 28, 2005

\begin{abstract}
The structural, dynamic, and thermodynamic properties of an excess electron interacting with an alkali cation $\left(\mathrm{Na}^{+}, \mathrm{K}^{+}, \mathrm{Li}^{+}\right)$in bulk water were investigated by means of a mixed quantum-classical molecular dynamics simulation technique. This study includes a reparametrization of the electron-cation pseudopotentials. The free energy calculations for all three systems show that a contact electron-cation pair can be observed, which is either as stable as the dissociated pair $\left(\mathrm{Li}^{+}\right)$or more stable by only a few $k T\left(\mathrm{Na}^{+}, \mathrm{K}^{+}\right)$. Given that the dissociation barrier is also quite small, we suggest that the average cation-electron distance in the experiments at room temperature will not depend on this free energy profile but rather on the minimization of the Coulombic repulsive interaction between like charges in the solvent medium. This enables us to compare the present molecular dynamics simulations with the spectroscopic data obtained for different ionic strengths. The overall trend of the UV-vis hydrated absorption spectra, namely, the shift toward shorter wavelengths at high ionic strengths, is fairly well reproduced. This confirms our hypothesis of statistical distribution of the cations and solvated electrons.
\end{abstract}

\section{Introduction}

Investigations of the structure, dynamics, and thermodynamics of the solvated electron in water are of great interest due to the importance of redox reactions in everyday physical chemistry and biophysics. The reactivity of the hydrated electron toward cations in solution has been extensively studied, mainly by pulse radiolysis. ${ }^{1,2}$ Noble metal ions such as $\mathrm{Ag}^{+}$are known to be easily reduced by the hydrated electron. ${ }^{3}$ On the other hand, alkaline metal cations $\left(\mathrm{M}^{+} ; \mathrm{M}=\mathrm{Li}, \mathrm{Na}, \mathrm{K}\right)$ do not react with the hydrated electron, since the $\mathrm{M}^{+} / \mathrm{M}$ couple has a lower redox potential than the hydrated electron. ${ }^{4-8}$ In such systems, water mediates the effective interactions between the ion and the excess electron and the formation of contact pairs was suggested. ${ }^{6,9,10}$ Electron spin-echo spectroscopy studies gave indirect information on the structure of the solvation shell of a solvated electron in alkaline glasses and ice. ${ }^{1-13}$ Recent resonance Raman spectra in bulk water supported these structural findings. ${ }^{14,15}$ Numerous investigations of the hydrated electron absorption spectrum were reported in the literature, under different ionic strength conditions and using various counterions. The overall trend through a systematic experimental study with different cations at various concentrations ${ }^{7,8}$ is the shift of the absorption of the hydrated electron toward shorter wavelengths ("blue shift").

The motivation for the present molecular simulation study is to provide a description, at the atomistic level, of the nature of the nonreactive alkali cation-solvated electron pairs in bulk water. A recently developed mixed quantum-classical molecular dynamics (QCMD) approach, ${ }^{16}$ based on an adiabatic simulation technique, is used to simulate an excess electron and a cation in bulk water. ${ }^{17-20}$ In this approach only one electron is treated quantum mechanically, while the effect of the surrounding molecules and cation is taken into account through effective pseudopotentials.

* To whom correspondence may be addressed. Fax: +331691561 88. E-mail: anne.boutin@lcp.u-psud.fr.
The paper is organized as follows. The models and methods are presented in the next section. In section 3, the force fields are described in some detail. A reparametrization of the electron-cation pseudopotentials is presented, and the new electron-sodium potential is tested against ab initio calculations of $\left[\mathrm{Na}^{+}, \mathrm{e}^{-},\left(\mathrm{H}_{2} \mathrm{O}\right)_{n}\right]$ clusters. The solvated electron absorption spectrum, free energy calculations, and structural information in the presence of a sodium cation are given in section 4 . Section 5 is devoted to a comparison between the sodium, lithium, and potassium cation behaviors. A simple model is suggested that enables an understanding of the general trend of the observed absorption blue shift in various ionic strength conditions.

\section{Simulation Techniques}

2.1. Adiabatic Mixed Quantum-Classical Molecular Dynamics. We perform a mixed quantum-classical molecular dynamics (QCMD) of an excess electron in bulk water, in the presence of different cations. While the water molecules and the cation are treated classically, the excess electron is treated quantum mechanically using the Born-Oppenheimer approximation. A given solvent/cation configuration $\mathbf{S}$ generates an effective interaction potential with the excess electron $\hat{V}_{\text {int }}(\mathbf{r}, \mathbf{S})$, where $\mathbf{r}$ denotes the electronic coordinates. The wave function describing the electron, $\psi_{n}(\mathbf{r}, \mathbf{S})$, and the corresponding energy, $E_{n}(\mathbf{S})$, are obtained by solving the stationary Schrödinger equation in this effective potential

$$
\left(\hat{T}_{\mathrm{e}}+\hat{V}_{\mathrm{int}}(\mathbf{r}, \mathbf{S})\right) \psi_{n}(\mathbf{r}, \mathbf{S})=E_{n}(\mathbf{S}) \psi_{n}(\mathbf{r}, \mathbf{S})
$$

where $\hat{T}_{\mathrm{e}}$ is the electronic kinetic energy operator. Equation 1 is solved by diagonalization for each solvent/cation configuration, i.e., at each time step. The Lánczos iterative algorithm (as implemented in the ARPACK library) was used to solve the electron eigenvalue problem, obtaining only the first 25 eigenstates for each time step. 
Once the eigenstates are known, the quantum contribution to the force acting on each classical particle is obtained by the Hellmann-Feynman theorem ${ }^{21,22}$

$$
\mathbf{F}_{\mathbf{S}, n}^{(\mathrm{q})}=-\nabla_{S} E_{n}(\mathbf{S})=-\left\langle\psi_{n}(\mathbf{r}, \mathbf{S})\left|\nabla_{S} \hat{V}_{\mathrm{int}}(\mathbf{r}, \mathbf{S})\right| \psi_{n}(\mathbf{r}, \mathbf{S})\right\rangle
$$

In the present study, all forces are computed for the ground state of the excess electron $(n=0)$. The wave functions $\psi_{n}(\mathbf{r}, \mathbf{S})$ are expanded into a basis of $7^{3}=343$ spherical Gaussian (stype) functions, centered on the nodes of a cubic lattice. The basis set parameters are identical to those used in ref 16 for the simulation of a hydrated electron: the Gaussian exponent $\alpha$ is $0.357 \AA^{-2}$ and the basis grid extension is $10 \AA$, which corresponds roughly to the half of the simulation box.

Standard periodic boundary conditions are used with the Ewald summation technique ${ }^{23}$ to calculate all classical and quantum long-range interactions. The simulations are performed in the NVT ensemble using the Nosé-Hoover thermostat ${ }^{24,25}$ to ensure isothermal conditions. The integration of the equations of motion for translation and rotation is performed using the Gear predictor-corrector algorithm, with a time step fixed at 0.5 fs. Simulations are run typically during a few tens of picoseconds.

2.2. Quantum Umbrella Sampling. Some of the simulations are performed with an electron/cation distance $\xi$ constrained around a given value $\xi_{0}$. This goal is achieved using the umbrella sampling approach, adding a quadratic term $U_{\xi_{0}}$ to the total Hamiltonian of the system

$$
U_{\xi_{0}}=\frac{1}{2} k\left(\xi(\mathbf{S})-\xi_{0}\right)^{2}
$$

Following the method proposed by Borgis and Staib, ${ }^{26}$ we adapted the umbrella sampling technique to a system containing a quantum particle. A new quantum umbrella sampling force acts on each classical atom, given by

$$
F_{\mathrm{i} \alpha}^{\mathrm{US}}=-\frac{\partial}{\partial S_{\mathrm{i} \alpha}} U_{\xi_{0}}=-k\left(\xi(\mathbf{S})-\xi_{0}\right) \frac{\partial \mathbf{r}(\mathbf{S})}{\partial S_{i \alpha}} \frac{\mathbf{r}(\mathbf{S})}{\xi(\mathbf{S})}
$$

where the nontrivial term $\partial \mathbf{r}(\mathbf{S}) / \partial S_{i \alpha}$ is calculated with a perturbative approach. ${ }^{26}$ The free energy profile along the electron-cation distance $\xi$ is given by

$$
F(\xi)=-k_{\mathrm{B}} T \ln P(\xi)
$$

where $P(\xi)$ is the probability to find the electron at distance $\xi$ from the cation

$$
P(\xi)=\frac{\left\langle\delta(r(\mathbf{S})-\xi) \exp \left(\beta U_{\xi_{0}}\right)\right\rangle_{\xi_{0}}}{\left\langle\exp \left(\beta U_{\xi_{0}}\right)\right\rangle_{\xi_{0}}}
$$

Umbrella sampling simulations are run for $50 \mathrm{ps}$, for $\xi_{0}$ values (see eq 3) ranging from 1 to $15 \AA$ with a $0.5 \AA$ increment. The $k$ value was fixed at $0.01 \mathrm{au} / \AA^{2}$, allowing fluctuations around $\xi_{0}$ of about $0.5 \AA$.

2.3. Absorption Spectra. Electron absorption spectra can be calculated by a direct average of the transition dipole moment or using the cumulant expansion and the vibration-rotation decorrelation hypothesis, ${ }^{26}$ leading to the formula

$$
I(E)=E\left(1-\mathrm{e}^{-\beta E}\right) \sum_{n=1}^{\infty}\left\langle\left|\mu_{0 n}\right|\right\rangle_{0} \exp \left[-\frac{\left(E-\left\langle E_{0 n}\right\rangle_{0}\right)^{2}}{\left\langle\delta E_{0 n}{ }^{2}\right\rangle_{0}}\right]
$$

TABLE 1: Lennard-Jones Parameters Used To Model the Water Oxygen/Cation Interactions, Taken from Ref 28

\begin{tabular}{lcll}
\hline & $\mathrm{Li}^{+}$ & \multicolumn{1}{c}{$\mathrm{Na}^{+}$} & \multicolumn{1}{c}{$\mathrm{K}^{+}$} \\
\hline$\epsilon_{i \mathrm{O}}(\mathrm{kJ} / \mathrm{mol})$ & 0.223 & $1.16 \times 10^{-2}$ & $1.37 \times 10^{-3}$ \\
$\sigma_{i \mathrm{O}}(\AA)$ & 0.385 & 3.33 & 4.74
\end{tabular}

where $E_{0 n} \equiv E_{0 n}(\mathbf{S})$ is the energy gap between the ground state and the $n$th excited state, whereas $\mu_{0 n} \equiv \mu_{0 n}(\mathbf{S})$ is the corresponding transition dipole. $\langle\cdots\rangle_{0}$ indicates an average over the solvent configurations with an electron equilibrated in its ground state. Both methods give similar absorption results; all spectra shown in this article are obtained by eq 7 .

\section{Force Fields and Pseudopotentials}

3.1. Classical Interaction Potentials. Water molecules and cations interact via classical interaction potentials consisting of a Coulombic and a 6-12 Lennard-Jones term. Water-water interactions are described by the SPC model, ${ }^{27}$ while cationwater interactions are described using parameters from $\AA$ qvist ${ }^{28}$ for alkaline cations. Water/cation interaction parameters are summarized in Table 1.

3.2. Interactions between the Electron and the Classical Particles. For the excess electron-water interaction, we use the pseudopotential developed by Turi and Borgis, ${ }^{29}$ based on quantum ab initio calculations for one water molecule plus an additional electron confined in a box in the static exchange theory limit. Details on the use of this pseudopotential with an excess electron described using a Gaussian grid basis set can be found in ref 16 .

The interactions between the excess electron and the cations are modeled using the one-electron semilocal pseudopotentials proposed by Durand and Barthelat ${ }^{30}$ for a cation $\mathrm{M}^{+}$

$$
\hat{V}_{\mathrm{e}-/ \mathrm{cat}}=-\frac{1}{r}+\hat{W}^{\mathrm{ps}}
$$

where $-1 / r$ is the Coulombic interaction term and $\hat{W}^{p s}$ is a nonlocal term. The analytical form of $\hat{W}^{\mathrm{ps}}$ is chosen to be

$$
\hat{W}^{\mathrm{ps}}(\mathbf{r})=\sum_{l} W_{l}(r) \hat{P}_{l}
$$

with

$$
W_{l}(r)=\exp \left(-\alpha_{l} r^{2}\right) \sum_{i=1}^{n_{l}} c_{i, l} r^{n_{i, l}}
$$

and

$$
\hat{P}_{l}=\sum_{m=-l}^{+l}|\operatorname{lm}\rangle\langle\operatorname{lm}|
$$

$\hat{P}_{l}$ is the projector on the spherical harmonics $Y_{l m}$ whose secondary quantum number is $l$. Computational details on the evaluation of these nonlocal terms using a Gaussian grid basis set can be found in ref 18. The original parameters of the nonlocal terms were optimized together with a basis set of s, p, and d Gaussians centered on the cation nucleus.

3.3. Reparametrization of the Electron/Cation Pseudopotentials. We have tested the electron/cation pseudopotentials on the isolated $\mathrm{M}^{0}$ atoms in a vacuum, using our $7^{3}$ Gaussian functions basis set. The energy levels for all $\left(\mathrm{M}^{+}+\mathrm{e}^{-}\right)$systems were compared to the experimental ionization potentials and excitation energies of $\mathrm{M}^{0}$. Neglecting the contribution due to the reorganization of the core orbitals, the ground-state energy 
TABLE 2: Ionization Potential (IP) and First Excitation Energy $(\Delta)$ Obtained Using Original and Optimized Pseudopotentials $^{a}$

\begin{tabular}{llccl}
\hline & & $\begin{array}{c}\text { pseudopotentials } \\
\text { (original, optimized) }\end{array}$ & exptl & $\begin{array}{c}\text { relative } \\
\text { errors }(\%)\end{array}$ \\
\hline $\mathrm{Li}$ & $\mathrm{IP}(\mathrm{eV})$ & $4.89,5.30$ & 5.39 & $9.4,1.6$ \\
& $\Delta(\mathrm{eV})$ & $1.57,1.90$ & 1.84 & $14.8,2.9$ \\
$\mathrm{Na}$ & $\mathrm{IP}(\mathrm{eV})$ & $4.76,5.11$ & 5.14 & $7.3,0.5$ \\
& $\Delta(\mathrm{eV})$ & $1.84,2.19$ & 2.10 & $12.5,4.1$ \\
$\mathrm{~K}$ & $\mathrm{IP}(\mathrm{eV})$ & $3.98,4.26$ & 4.34 & $8.3,1.7$ \\
& $\Delta(\mathrm{eV})$ & $1.51,1.65$ & 1.61 & $6.3,2.6$
\end{tabular}

${ }^{a}$ These results are compared to the experimental values from ref 40 .

$\epsilon_{0}$ of the excess electron can be related to the ionization potential of $\mathrm{M}^{0}$ through $\epsilon_{0}=-\mathrm{IP}$.

For all cations, the original pseudopotentials reproduce the desired experimental properties (ionization potential and first excitation energies) within a $15 \%$ range, as reported in Table 2. The differences observed are attributed to the different basis set used as well as to the lack of core-valence correlation and core polarization. Thus, for each cation a new set of parameters was designed by minimization of the difference between calculated and experimental properties. In this article, these reparametrized potentials will be called optimized pseudopotentials. Due to the functional form of the nonlocal term $\hat{W}^{\mathrm{ps}}(\mathbf{r})$, a separate optimization of the $\mathrm{s}, \mathrm{p}$, and $\mathrm{d}$ parts of the pseudopotentials, corresponding to $W_{0}(r), W_{1}(r)$, and $W_{2}(r)$ in eq 9 , was possible.

Taking into account the ionization potential and the energy of the first $\mathrm{s} \rightarrow \mathrm{p}$ and $\mathrm{s} \rightarrow \mathrm{d}$ transitions, we defined the relative root mean square (RRMS) error for each part of the pseudopotential

$$
\begin{gathered}
\operatorname{RRMS}(s)=\left|\frac{\mathrm{IP}-\mathrm{IP}_{\exp }}{\mathrm{IP}_{\exp }}\right| \\
\operatorname{RRMS}(p)=\left|\frac{\Delta E^{n \mathrm{~s} \rightarrow n \mathrm{p}}-\Delta E_{\mathrm{exp}}^{n s \rightarrow n \mathrm{p}}}{\Delta E_{\exp }^{n s \rightarrow n \mathrm{p}}}\right| \\
\operatorname{RRMS}(d)=\left|\frac{\Delta E^{n \mathrm{~s} \rightarrow n \mathrm{~d}}-\Delta E_{\mathrm{exp}}^{n s \rightarrow n \mathrm{~d}}}{\Delta E_{\exp }^{n s \rightarrow n d}}\right|
\end{gathered}
$$

where IP is the first ionization potential of $\mathrm{M}^{0}$ and $\Delta E^{i \rightarrow j}$ is the transition energy between electronic states $i$ and $j$ of $\mathbf{M}^{0}$, while the "exp" subscript denotes the experimental value of a given physical quantity.

We found many minima for each RRMS and chose parameters close to the original ones yielding small RRMS values. The optimized parameters for $\mathrm{Li}^{+}, \mathrm{Na}^{+}$, and $\mathrm{K}^{+}$cations can be found in the Appendix A, as well as the original parameters. In vacuo properties for $\mathrm{Li}, \mathrm{Na}$, and $\mathrm{K}$ with these new parameters are in better agreement with the experimental data, as shown in Table 2.

3.4. Validation of the Electron/Cation Pseudopotentials. The electron/cation pseudopotentials have been adjusted and reoptimized for the isolated neutral atoms properties. To check their ability to fairly describe the cation-electron pair (i.e., when the electron is localized outside the cation), the electron-sodium pseudopotentials have been compared to ab initio calculations on sodium-electron pair systems. Several configurations have been selected from QCMD simulation runs. We have retained a few water molecules around the electron-cation pair thus

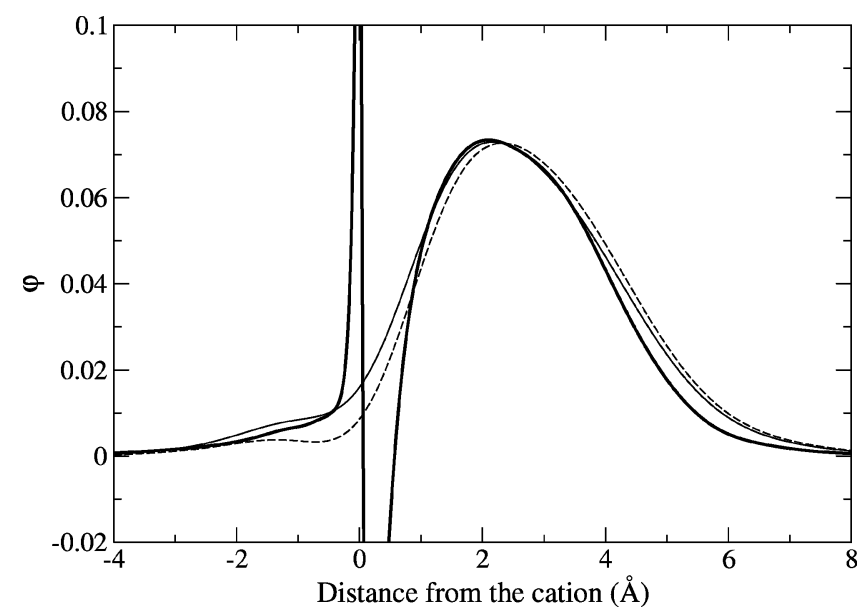

Figure 1. Comparison of the ab initio orbital (thick line) and pseudoorbitals of the ground state using both the original (dashed line) and the optimized (solid line) parameter sets of the electron-cation pseudopotential in the case of the electron-sodium pair solvated by 28 water molecules in a vacuum. The value of the orbital is plotted along the axis joining the cation and the center of the electronic density.

TABLE 3: Comparison of TDSCF and MCQDPT Transition Energies (in eV) and TDSCF Oscillator Strengths on a $\left(\mathrm{H}_{2} \mathrm{O}\right)_{5}{ }^{-}$Configuration

\begin{tabular}{cccc}
\hline transition & $\begin{array}{c}\text { MCQDPT } \\
\text { transition energy }\end{array}$ & $\begin{array}{c}\text { TDSCF } \\
\text { transition energy }\end{array}$ & $\begin{array}{c}\text { TDSCF } \\
\text { oscillator strength }\end{array}$ \\
\hline $0 \rightarrow 1$ & 1.105 & 0.979 & 0.324 \\
$0 \rightarrow 2$ & 1.296 & 1.172 & 0.313 \\
$0 \rightarrow 3$ & 1.647 & 1.513 & 0.110 \\
$0 \rightarrow 4$ & 1.814 & 1.678 & 0.224
\end{tabular}

generating free $\left(\mathrm{Na}^{+}, \mathrm{e}^{-},\left(\mathrm{H}_{2} \mathrm{O}\right)_{n}\right)$ clusters with $n$ varying from 14 to 99 . We have used the TDSCF $^{31}$ method implemented in the Gaussian 03 package. ${ }^{32}$ The Gaussian basis sets used for these ab initio calculations are given in the Appendix B. This crude TDSCF (time-dependent self-consistent field) calculation has first been compared to a high-level MCQDPT (multiconfigurational quasi-degenerate perturbation theory) calculation on a much smaller cluster of five water molecules $\left(\mathrm{H}_{2} \mathrm{O}\right)_{5}{ }^{-}$using the MCQDPT method of Nakano ${ }^{33}$ implemented in the GAMESS 2003 package. ${ }^{34}$ The results are given in Table 3. The TDSCF transition energies are only slightly smaller than the MCQDPT ones (by $\simeq 0.1 \mathrm{eV}$ ). The core-valence correlation, neglected in TDSCF, is small in such systems. The TDSCF method thus appears reasonable in this case.

We present here a comparison between the pseudopotentials approach and the TDSCF one on the $\left(\mathrm{Na}^{+}, \mathrm{e}^{-},\left(\mathrm{H}_{2} \mathrm{O}\right)_{28}\right)$ system. As shown in Table 4, the transition energies obtained by the TDSCF and by the two pseudopotentials are very close to each other. To discriminate which of the original and optimized pseudopotentials is the most realistic one, we have compared the shape of the ground-state orbital along the axis joining the $\mathrm{Na}$ nucleus and the excess electron barycenter (see Figure 1). Since the use of pseudopotentials smooths out the rapid oscillation of the ab initio orbital in the vicinity of the $\mathrm{Na}$ nucleus, the comparison between orbital shapes must be made at distance from the cation larger than $1 \AA$. Figure 1 shows that the orbital obtained using the optimized pseudopotential is closer to the ab initio one, especially in the high probability density region. This shows that on top of ameliorating the $\mathrm{Na}$ atom spectroscopy, the reoptimization of the pseudopotential also improves the description of the cation-electron pair. 
TABLE 4: One Electron Solvated by $1 \mathrm{Na}^{+}$and $28 \mathrm{H}_{2} \mathrm{O}$ Molecules: Comparison of TDSCF and QCMD Transition Energies (in eV) and Oscillator Strengths on a $\mathrm{Na}\left(\mathrm{H}_{2} \mathrm{O}\right)_{28}{ }^{+}$Configuration

\begin{tabular}{cccccc}
\hline & $\begin{array}{c}\text { TDSCF } \\
\text { transition } \\
\text { energy }\end{array}$ & $\begin{array}{c}\text { TDSCF } \\
\text { oscillator } \\
\text { strength }\end{array}$ & $\begin{array}{c}\text { original } \\
\text { pseudotransition } \\
\text { energy }\end{array}$ & $\begin{array}{c}\text { optimized } \\
\text { pseudotransition } \\
\text { energy }\end{array}$ & $\begin{array}{c}\text { optimized } \\
\text { pseudooscillator } \\
\text { strength }\end{array}$ \\
\hline $0 \rightarrow 1$ & 2.274 & 0.289 & 2.295 & 2.316 & 0.335 \\
$0 \rightarrow 2$ & 2.545 & 0.247 & 2.390 & 2.406 & 0.330 \\
$0 \rightarrow 3$ & 2.760 & 0.235 & 2.724 & 2.766 & 0.334
\end{tabular}

\section{The Sodium Cation}

4.1. Influence of the Pseudopotential Parametrization and Finite Size Effects. As a first step in this work, we investigated the influence of the pseudopotential parameters describing the electron/cation interaction on the thermodynamic results, as well as the influence of the size of the simulation box. Structural, spectroscopic, and thermodynamic information on this system was obtained by the mixed quantum-classical technique described earlier, using the two different sets of parameters for the electron/cation pseudopotential discussed in the previous section and two different sizes for the simulation box. The two different cubic simulation boxes used contained 300 and 800 water molecules, one sodium cation, and an excess electron. The simulations were performed at temperature $T=298 \mathrm{~K}$, with a density of $\rho=1 \mathrm{~g} \cdot \mathrm{cm}^{-3}$.

The free energy profiles obtained using both sets of pseudopotential parameters are shown in Figure 2, for a system containing the sodium cation, an excess electron, and 800 water molecules. In both cases, there is a local minimum of the free energy profile at short distances, corresponding to an electroncation contact pair, ${ }^{18,19}$ and a free energy barrier separating this state from the state where the electron and the cation are separately solvated (large electron-cation distance). But while both sets of parameters for the electron/cation pseudopotential exhibit this same general behavior, they yield quantitatively different results. It can be seen that the simulations using the optimized pseudopotential exhibit a higher free energy barrier between the two states (approximately $6 \mathrm{kT}$, compared to $3 \mathrm{kT}$ for the original parameters), as well as a shorter equilibrium distance for the electron-cation contact pair (around $1.7 \AA$, compared to $2.2 \AA$ for the original pseudopotential). This can be related to the fact that the optimized pseudopotential gives a larger value of the ionization potential for the sodium atom

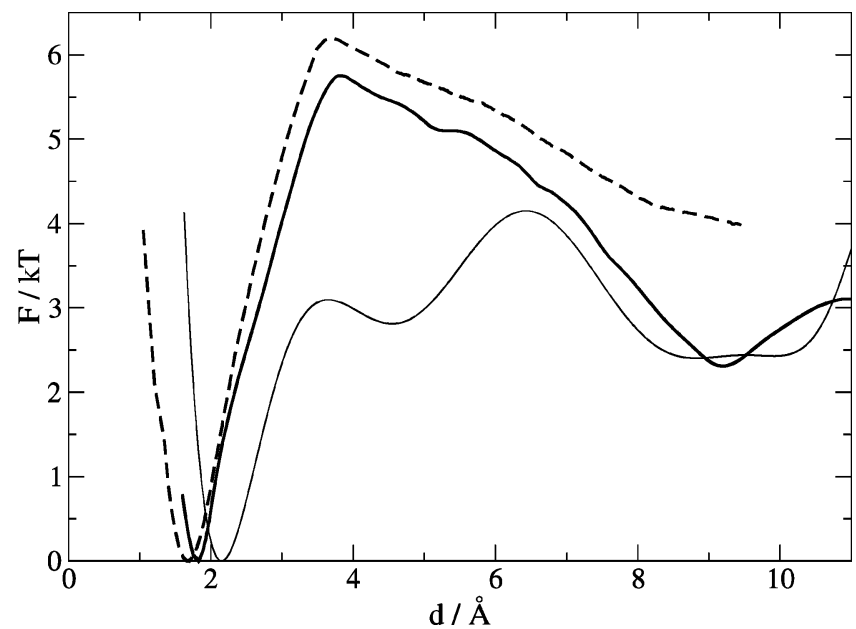

Figure 2. Free energy profiles as a function of the electron-sodium distance obtained using the original pseudopotential (thin solid line) as well as the optimized set of parameters (thick solid line) for a system containing 800 water molecules. The free energy profile obtained with the optimized pseudopotential and 300 water molecules is plotted as a dashed line. in a vacuum than the original parameters (see Table 2), thus better reproducing the stability of the electron in the vicinity of the sodium cation. On the basis of this interpretation, we suggest that the free energy curve using the optimized pseudopotential is closer to reality.

The dependence of the size of the simulation box on the thermodynamic and structural properties of the sodium-electron system in bulk water was also studied. Figure 2 also shows the free energy profiles as a function of the electron-sodium distance for systems containing 300 and 800 water molecules. It can be seen that the size of the simulation box has little influence on the equilibrium distance for the contact pair. Indeed, radial density functions (not presented here) for the sodiumelectron contact pair in systems containing 300 and 800 water molecules are very similar, indicating that the behavior of this system at short distances is not much influenced by finite-size effects. The thermodynamic information concerning the large electron-cation distances depends strongly on the size of the system chosen for the study, preventing us from drawing definite conclusions about the relative stability of the short- and longdistance states. However, it is interesting to note that the shortdistance electron-sodium pair seems to be more stable than the separately solvated species for systems containing 800 water molecules by some $2 k T$.

The unconstrained dynamics of the electron-sodium system solvated in water can also shed some light on this important question of the relative stability of the contact pair and the separately solvated species. The evolution of the electronsodium distance during unconstrained simulations is reported in Figure 3, starting from four different configurations with an initial electron-cation distance between 5 and $7 \AA$. It can be seen that three of these trajectories lead to the electron-sodium pair within times ranging from 2 to $10 \mathrm{ps,} \mathrm{while} \mathrm{the} \mathrm{fourth}$ simulation does not exhibit electron-cation distances smaller than $5 \AA$. The fact that we observe a spontaneous formation of the contact pair for three systems while the reverse process never occurs in the time scale of 100 ps supports the conclusion that

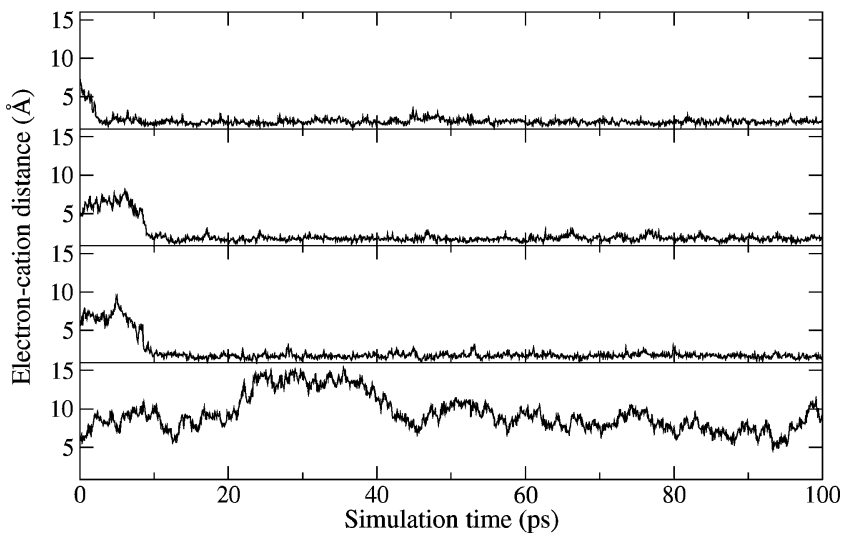

Figure 3. Electron-cation distance as a function of simulation time during unconstrained simulations of $\mathrm{Na}^{+}$and an excess electron with 300 water molecules, starting from four different initial configurations. Data obtained using the optimized set of parameters for the electron/ sodium pseudopotential. 


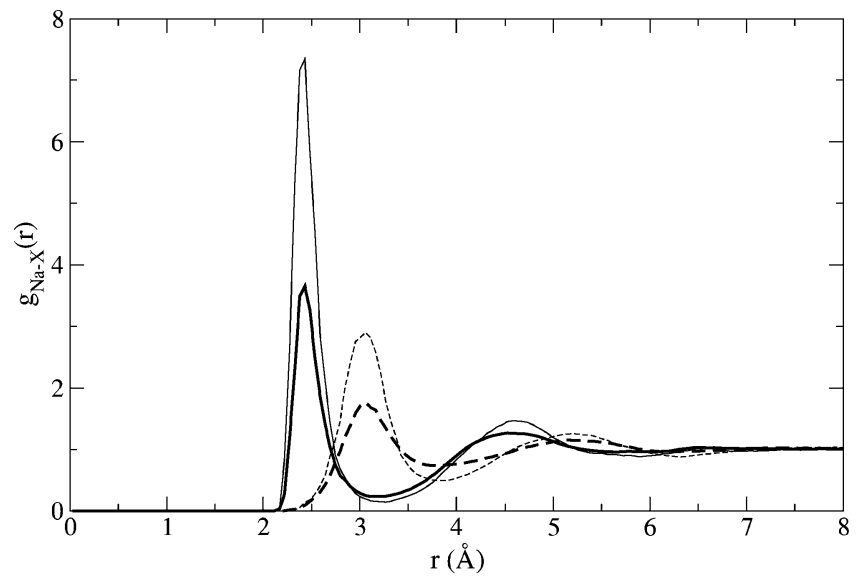

Figure 4. Radial density functions for the distribution of oxygen (solid line) and hydrogen (dashed line) atoms around the sodium cation in the $\left(\mathrm{Na}^{+}, \mathrm{e}^{-}\right)$contact pair (thick lines). The radial density functions of $\mathrm{Na}-\mathrm{O}$ and $\mathrm{Na}-\mathrm{H}$ for a single sodium cation in pure water are shown for comparison (thin lines).

the electron-sodium pair is the most stable state in our simulation model. It seems clear, however, that in the long time scale (thermodynamic limit) an equilibrium between the contact pair and the dissociated solvated species will take place at room temperature. This is because the free energy difference between these two states is on the order of $k T$, and the free energy barrier is smaller than $10 \mathrm{kT}$.

4.2. Structural Information. In this section, we present structural information about the electron-sodium contact pair and its solvation shell. The radial density functions (RDFs) for the sodium-oxygen and sodium-hydrogen pairs are presented in Figure 4, for both the sodium-electron pair and the single sodium cation in bulk water. The main solvation features are found to be the same in both cases, like the first maximum of the $\mathrm{Na}-\mathrm{O} \mathrm{RDF}$ at $r \approx 2.4 \AA$ and its first minimum at $r \approx 3.2$ $\AA$. The main difference between these two cases is the relative height of the peaks, yielding a number of water molecules in the first solvation shell of 3.4 for the electron-cation pair, to be compared with the 5.9 water molecules present in the first solvation shell of the single sodium cation in bulk water. Thus, the structure of the solvation shell of the $\mathrm{Na}^{+}$cation is unchanged in the presence or absence of hydrated electron. The difference in the number of water molecules in the first solvation shell can simply be accounted for by the volume occupied by the hydrated electron.

On the contrary, electron-oxygen and electron-hydrogen RDFs in the sodium-electron pair are very different from those of the solvated electron in bulk water (see Figure 5). The first peak in the electron-hydrogen radial distribution function is located at 2.1 and $2.2 \AA$ for the solvated electron and sodiumelectron pair in bulk water, respectively. The MD simulation reveals that the water molecules are strongly oriented toward the electron to form hydrogen bonds. The number of water molecules in the first solvation shell is found to be 4.2 in the case of the hydrated electron and drops to 2 for the solvated sodium-electron contact pair. Moreover, we find that the solvation structure is less clear, indicating that the solvation of the electron in the pair is weaker than that of the electron in bulk water.

Experimental structural studies have mainly been performed at very low temperature on glasses and ice systems. In $\gamma$-irradiated $10 \mathrm{M} \mathrm{NaOH}$ alkaline aqueous glasses, analysis of electron spin-echo data, which first leads to the octahedral model, ${ }^{11,35,36}$ suggests that two bond-oriented water molecules

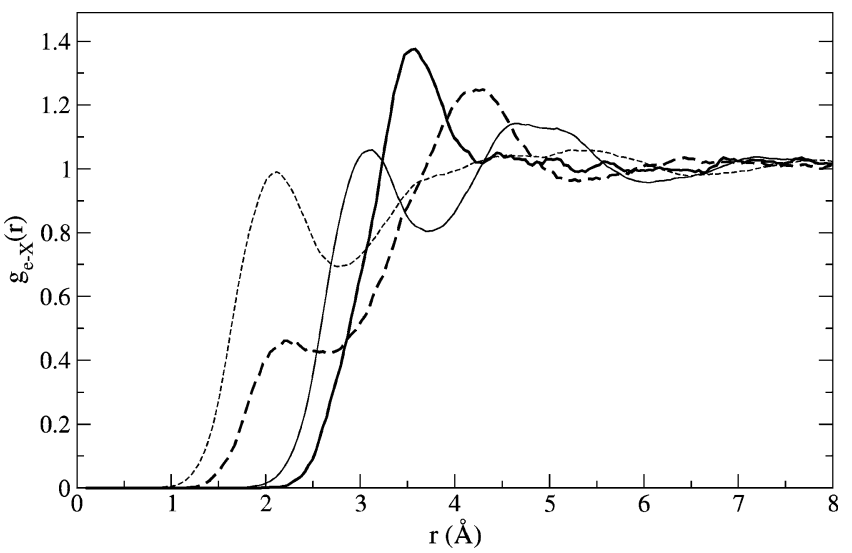

Figure 5. Radial density functions for the distribution of oxygen (solid line) and hydrogen (dashed line) atoms around the center of electronic density for the excess electron in the $\left(\mathrm{Na}^{+}, \mathrm{e}^{-}\right)$contact pair (thick lines). The radial density functions corresponding to oxygen an hydrogen around a solvated electron in bulk water are shown for comparison (thin lines).

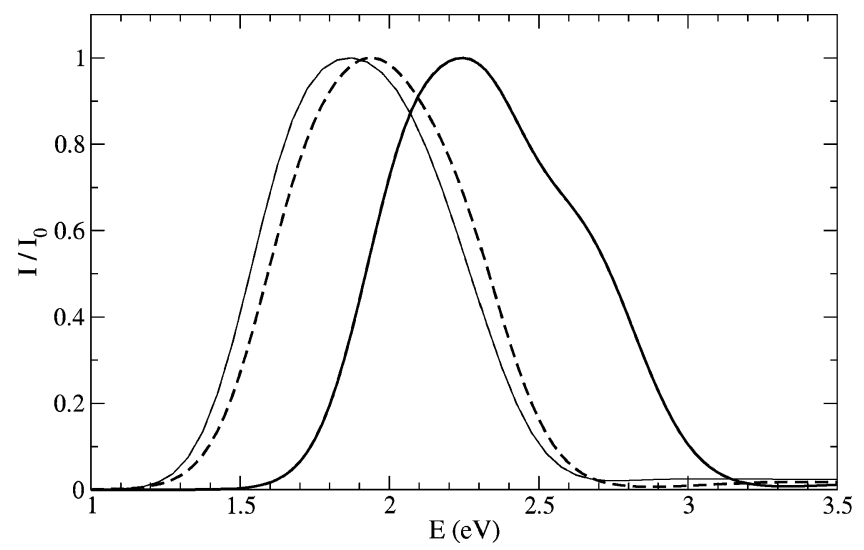

Figure 6. Normalized absorption spectra of the solvated electron with a sodium cation, for the contact pair (thick solid line) and separately solvated species (dashed line). The absorption spectrum for an hydrated electron without cation is also shown for comparison (thin solid line).

are located at $2.1 \AA$ from the electron center. ${ }^{12,13}$ Our simulation data agree well with these experimental findings and strongly support that the two hydrogen atoms of the first solvation peak do not belong to the same molecule. This hydrogen bonding like interaction has recently been confirmed by Raman experiments. ${ }^{14,15}$

4.3. Absorption Spectrum. The UV-vis absorption spectra of the solvated electron with a sodium cation and 300 SPC water molecules at ambient conditions are shown in Figure 6, for both the separately solvated species and the electron-sodium contact pair. The presence of the cation induces a blue shift of the absorption spectrum of the solvated electron. In the case of the separately solvated species, the maximum of the absorption spectrum is located at $E=1.95 \mathrm{eV}$, which represents a shift of $0.07 \mathrm{eV}$ from the solvated electron in bulk water, while the shape of the band and its half-maximum width are unchanged. An analysis of the contributions to the total electron-cation interaction shows that such long-distance influence is indeed purely Coulombic. The absorption spectrum of the electron in the contact electron-sodium pair is located at much higher energy (2.25 eV) and exhibits a slightly different band shape, with a shoulder on its high-energy wing and a half-maximum width $+0.1 \mathrm{eV}$ larger than that of the solvated electron. The large shift at small electron-cation distances is mainly explained by the stronger Coulombic interaction between the electron and the cation. Moreover, previous work showed that the change in 


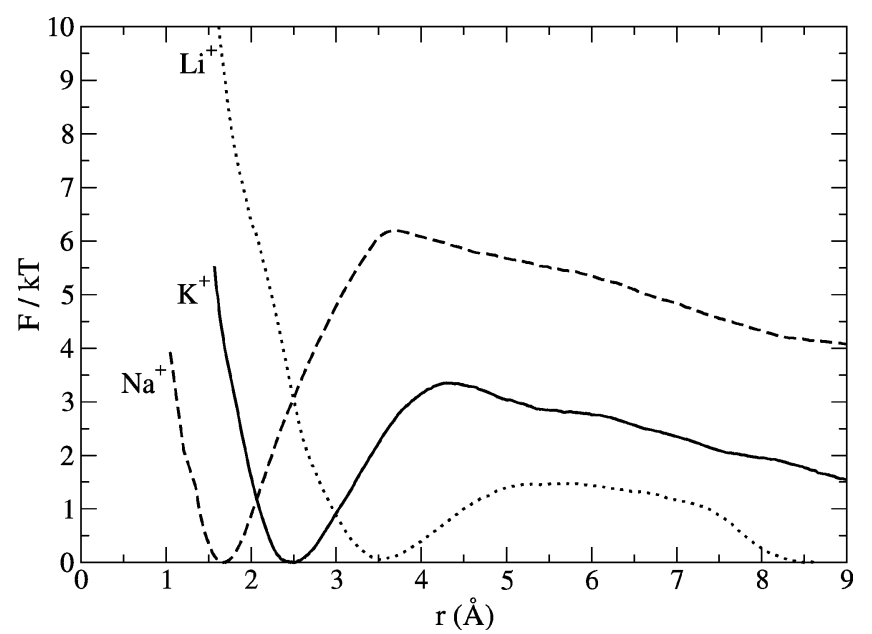

Figure 7. Free energy profiles as a function of electron-cation distance obtained for lithium (dotted line), sodium (dashed line), and potassium (solid line) simulations.

the shape of the spectrum and the existence of a shoulder are due to one of the three p-like excited states of the solvated electron (the one with $\Sigma$ symmetry, its orbital pointing in the direction of the cation) being destabilized by core repulsion. ${ }^{18}$ A similar phenomenon has been reported experimentally in the case of earth alkaline cations and an excess electron in THF. ${ }^{10,37}$ Furthermore, the presence of this shoulder on the spectrum of the solvated electron near a sodium cation has been reported in an experimental study by Gelabert and Gauduel. ${ }^{9}$

Comparing the spectroscopic information obtained from simulation to experimental data is not straightforward due to the importance of many factors such as the concentration of ions and the presence of different counterions. ${ }^{4-8}$ The concentration effect will be discussed below.

\section{Other Cations}

To study the behavior of the hydrated electron in the presence of different alkali cations, simulations were performed on systems containing one cation $\left(\mathrm{Li}^{+}\right.$or $\left.\mathrm{K}^{+}\right), 300$ water molecules, and an excess electron. For each system, we performed free energy calculations using quantum umbrella sampling as well as $100 \mathrm{ps}$ of unconstrained simulations starting from four different initial configurations. All simulations were done with sets of parameters for the electron-cation pseudopotential optimized as described in section 3.3. The results obtained are compared with the case of the sodium cation presented in detail in the previous section.

5.1. Free Energy Profiles. The free energy profiles as a function of the electron-cation distance $r$ for the three alkali cations $\left(\mathrm{Li}^{+}, \mathrm{Na}^{+}, \mathrm{K}^{+}\right)$are shown in Figure 7 . For all three cations, these profiles exhibit two local minima, at short distance
TABLE 5: Main Characteristics of the Absorption Spectra for Alkali-Electron Systems: Position of the Maximum $\left(E_{\max }\right)$ and Half-Maximum Width $\left(\Delta E_{1 / 2}\right)^{a}$

\begin{tabular}{lccccccc}
\hline & & & \multicolumn{2}{c}{$\mathrm{Na}^{+}-\mathrm{e}^{-}$} & & \multicolumn{2}{c}{$\mathrm{K}^{+}-\mathrm{e}^{-}$} \\
& $\mathrm{e}^{-}$ & $\mathrm{Li}^{+}-\mathrm{e}^{-}$ & sep & pair & & sep & pair \\
\hline$E_{\max }(\mathrm{eV})$ & 1.87 & 1.94 & 1.94 & 2.25 & & 1.93 & 2.02 \\
$\Delta E_{1 / 2}(\mathrm{eV})$ & 0.74 & 0.75 & 0.73 & 0.82 & & 0.74 & 0.84
\end{tabular}

${ }^{a}$ The characteristics of the spectrum of a hydrated electron without cation are indicated for comparison.

$(r<4 \AA)$ and at infinite distance. The height of the free energy barrier separating these minima decreases from the sodium cation $(6.2 \mathrm{kT})$ to the potassium $(3.3 \mathrm{kT})$ and the lithium cation $(1.5 k T)$. The time evolution of the electron-cation distance during unconstrained simulations of systems containing a potassium cation, presented in Figure 8 (left panel), reveals the existence of two metastable states clearly separated. A few crossings of the free energy barrier are observed on the time scale of 100 ps. However, unlike $\mathrm{Na}^{+}$, some crossings of the barrier in the case of $\mathrm{K}^{+}$lead to the separately solvated species. This event is not frequent enough during our simulations to draw a definite conclusion upon the stability of the electronpotassium pair. It is important to note that, due to the finite size of our simulation box $(\approx 21 \AA)$, free energy information obtained is limited to distances smaller than $9-10 \AA$. In the case of the lithium cation, the free energy barrier is approximately $1.5 k T$, and unconstrained simulations exhibit frequent crossings of this barrier (see Figure 8, right panel). No stable $\mathrm{Li}^{+}$-electron pair was observed during our simulations.

We suggest that the existence of an electron-cation pair for $\mathrm{Na}^{+}$and $\mathrm{K}^{+}$can be related to their standard reduction potential, higher than that of $\mathrm{Li}^{+}: E_{\mathrm{Na}^{+} / \mathrm{Na}}^{\circ} \simeq-2.71 \mathrm{~V}$ while $E_{\mathrm{Li}^{+} / \mathrm{Li}}^{\circ} \simeq$ $-3.04 \mathrm{~V}$ and $E_{\mathrm{K}^{+} / \mathrm{K}}^{\circ} \simeq-2.93 \mathrm{~V} .{ }^{38}$ This can be interpreted as a stronger ability of the sodium cation, and to a lesser extent of the potassium cation, to accommodate an electron in its close neighborhood, explaining the formation of stable $\left(\mathrm{Na}^{+}, \mathrm{e}^{-}\right)$and $\left(\mathrm{K}^{+}, \mathrm{e}^{-}\right)$contact pairs.

5.2. Electron Absorption Spectra. The UV-vis absorption spectra of an alkali cation plus an excess electron in 300 SPC water molecules at ambient conditions are shown in Figure 9, and their main characteristics are reported in Table 5. The absorption spectra were obtained by averaging over 85 ps simulations starting from four different initial configurations (after a 15 ps equilibration run). For $\mathrm{Na}^{+}$and $\mathrm{K}^{+}$cations, the separately solvated species and the electron-cation pair states were separately averaged and the spectra of both metastable states are shown. The effect of the cation on the absorption band of the hydrated electron is similar for $\mathrm{Li}^{+}$and $\mathrm{K}^{+}$to the effect observed for the sodium cation. However, the maximum of the spectrum for the potassium-electron pair is located at a lower

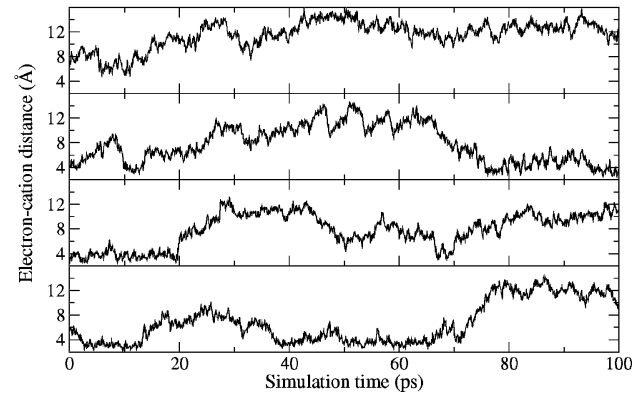

Figure 8. Electron-cation distance as a function of simulation time during unconstrained simulations of $\mathrm{K}^{+}$(left panel) and $\mathrm{Li}^{+}$(right panel) and an excess electron with 300 water molecules. 


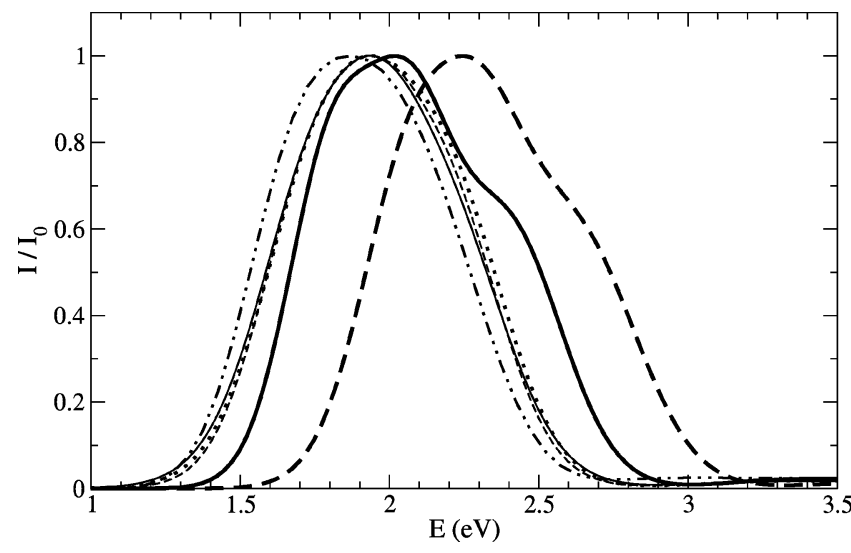

Figure 9. Normalized absorption spectra for the solvated electron with different alkali cations: $\mathrm{Li}^{+}$(dotted line), $\mathrm{Na}^{+}$(dashed line), and $\mathrm{K}^{+}$ (solid line). For $\mathrm{Na}^{+}$and $\mathrm{K}^{+}$, two spectra are shown: the contact electron-cation pair (thick line, at higher energies) and the separately solvated species (thin line, at lower energies); for details, see section 5.2. The absorption spectrum for a hydrated electron without cation is also shown (dash-dot-dot line).

energy than that of the sodium-electron pair, due to the larger electron-cation distance in the contact pair.

5.3. A Simple Model for the Ionic Strength Dependence of the Absorption Spectra. As stated in the Introduction section, several hydrated electron absorption studies have been reported in the literature, using various ionic strength conditions. A way to compare these data with the present MD simulations is to consider the following simple model. The free energy calculations for all three electron-ion systems (sodium, lithium, and potassium) clearly show that the contact electron-cation pair is either as stable as the dissociated pair or more stable by only a few $k T$. Given that the dissociation barrier is also quite small, the average cation-electron distance $d$ in the experiments will presumably hardly depend on the above-mentioned free energy profile but rather on the minimization of the Coulombic repulsive interaction between like charges in the solvent medium. The alkali cations are thus considered to be distributed statistically around the solvated electron. This allows relation of the average cation-electron distance $d$ directly to the cation concentration $C$ through

$$
d=\frac{1}{2\left(C N_{\mathrm{A}}\right)^{1 / 3}}
$$

where $N_{\mathrm{A}}$ is Avogadro's number.

The hypothesis of randomly arranged cations with respect to trapped electrons has already been put forward by electron spinecho analysis of various $\mathrm{Na}$ concentrations of alkaline glasses. ${ }^{12,13}$ For a $10 \mathrm{M} \mathrm{NaOH}$ glass, the electron-sodium distance is estimated to $2.6-2.9 \AA$. Our simple model predicts a distance of $2.7 \AA$ for a sodium concentration of $10 \mathrm{M}$. This model enables us to compare the experimental absorption data obtained for different ionic strengths with the present MD simulations for various constrained electron-cation distances. The experimental absorption shifts due to the presence of cations, $\Delta E$, are reported in Figure 10 together with the MD results. In this figure, the alkali-cation concentrations were converted to average cationelectron distances using eq 15 . The experimental and simulated spectral shifts were determined by the difference of the absorption spectrum maxima of the solvated electron in the concentrated salt solution and in pure water at the same temperature obtained experimentally and by simulation, respectively. This takes into account the fact that experiments have been performed at various temperatures.

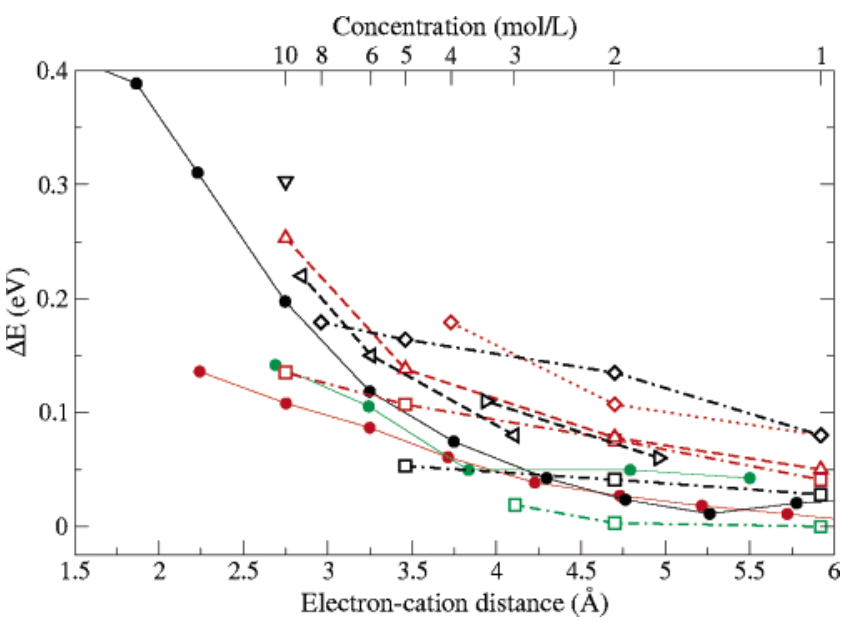

Figure 10. Energy shift of the absorption band maximum of the solvated electron with different cations $\left(\mathrm{Li}^{+}\right.$, red; $\mathrm{Na}^{+}$, black; $\mathrm{K}^{+}$, green) as a function of the electron-cation distance. Simulations results from this work (solid lines) are compared to experimental results from refs 5 ( $\mathrm{LiCl}$, triangle up), $6\left(\mathrm{Na}_{2} \mathrm{SO}_{4}\right.$, triangle left, and $\mathrm{NaClO}_{4}$, triangle right), $8\left(\mathrm{NaCl}\right.$ and $\mathrm{LiCl}$, square, and $\mathrm{NaClO}_{4}$, diamond), and 41 (triangle down) using the concentration-distance relation from eq 15.

The simulation results reveal that a significant shift is observed only at short distance, lower than 5-6 $\mathrm{A}$. A cation effect is obtained on the absorption spectrum if the first solvation shell of the hydrated electron is modified. The absorption spectrum of a solvent-separated cation-electron pair is found to be similar to the single hydrated electron one. This is consistent with the fact that a shift of the absorption spectra is only observed at high salt concentration experimentally. We observed for all three cations a blue shift of the same order of magnitude as in experiments. Given the crudeness of the concentration-distance relation used, the agreement can be considered as fair. It seems clear that a more detailed interpretation of the experiments will have to consider the effect of the counterions in the simulations. Whether or not the salts are fully dissociated in the experiments at high ionic strength would also have to be carefully studied.

\section{Conclusions}

We have performed MD simulations of an excess electron in bulk water in the presence of $\mathrm{Li}^{+}, \mathrm{Na}^{+}$, and $\mathrm{K}^{+}$cations in bulk water at ambient conditions. We have developed new one electron pseudopotential parameters for describing alkali cationselectron interactions based on ab initio calculation. In the case of the sodium cation, we observe a contact pair which is more stable than the dissociated pair by a few $k T$. We have carefully checked the effect of the pseudopotential parameters and the system size and found that the existence of this contact pair is not sensitive to our model parameters. For the potassium cation, the contact electron-cation pair is also a stable state but the free energy barrier is lower than that in the case of sodium. We observed spontaneous crossings of the barrier leading to the formation or the dissociation of the electron-cation pair in the MD time scale. In the case of lithium, the more stable state is found to be the separated solvated species. The free energy barriers are however small in all cases, and we predict that the equilibrium electron-cation distance in bulk water will not be dominated by the free energy profile.

The structure of the first solvation shell of the solvated electron in the presence of a sodium cation in bulk water, i.e., two hydrogen bonding water molecules, has been found to be in agreement with electron spin-echo analysis. 
TABLE 6: Parameters of the Original Pseudopotentials (in atomic units)

\begin{tabular}{|c|c|c|c|c|c|c|c|c|c|c|c|}
\hline \multicolumn{4}{|c|}{$\mathrm{Li}^{+}$} & \multicolumn{4}{|c|}{$\mathrm{Na}^{+}$} & \multicolumn{4}{|c|}{$\mathrm{K}^{+}$} \\
\hline$l$ & $\alpha$ & $n_{i, l}$ & $c_{i, l}$ & $l$ & $\alpha$ & $n_{i, l}$ & $c_{i, l}$ & $l$ & $\alpha$ & $n_{i, l}$ & $c_{i, l}$ \\
\hline 0 & 1.04883 & -2 & 3.48672 & 0 & 1.33118 & 0 & 9.90386 & 0 & 0.67329 & 0 & 6.87490 \\
\hline & & 2 & 0.49988 & 1 & 0.60152 & 0 & 1.79437 & 1 & 0.33264 & 0 & 2.06028 \\
\hline 1 & 1.40580 & -1 & -0.77469 & 2 & 0.98792 & 0 & -2.39927 & 2 & 0.78996 & 0 & -6.24321 \\
\hline
\end{tabular}

TABLE 7: Parameters of the Optimized Pseudopotentials (in atomic units)

\begin{tabular}{|c|c|c|c|c|c|c|c|c|c|c|c|}
\hline \multicolumn{4}{|c|}{$\mathrm{Li}^{+}$} & \multicolumn{4}{|c|}{$\mathrm{Na}^{+}$} & \multicolumn{4}{|c|}{$\mathrm{K}^{+}$} \\
\hline$l$ & $\alpha$ & $n_{i, l}$ & $c_{i, l}$ & $l$ & $\alpha$ & $n_{i, l}$ & $c_{i, l}$ & $l$ & $\alpha$ & $n_{i, l}$ & $c_{i, l}$ \\
\hline 0 & 1.46836 & -2 & 2.44070 & 0 & 1.59742 & 0 & 8.91347 & 0 & 0.80795 & 0 & 6.87490 \\
\hline & & 2 & 0.49988 & 1 & 0.66167 & 0 & 1.79437 & 1 & 0.39917 & 0 & 2.06028 \\
\hline 1 & 0.84348 & -1 & -1.08457 & 2 & 0.98792 & 0 & -2.39927 & 2 & 0.47397 & 0 & -6.86753 \\
\hline
\end{tabular}

The calculated absorption spectrum is dominated by the solvated electron-cation distance. When the electron-cation distance decreases, the absorption spectrum is shifted to higher energy. This is good agreement with experimental evidence of absorption spectrum blue shift with increasing cation concentration. We suggest a relation between the cation concentration and the equilibrium solvated electron-cation distance based on a random cation distribution. This model enables us to compare our single cation simulations with experiments at high salt concentration. We found a qualitatively good agreement between simulations and experiments which supports the conclusion that the equilibrium solvated electron-cation distance is dominated by the minimization of the Coulombic repulsive interaction between like charges in the solvent medium.

In this work, we have limited ourselves to simulations with only one cation in the simulation box. At high salt concentration, the average distance between cations is quite small (lower than $6 \AA$ ) and the interaction between cations has to be explicitly taken into account to improve the simulation model. To have a better insight of the salt concentration effect, simulation work on systems containing more than one cation and explicit counterions is in progress.

Acknowledgment. We thank the Institut du Développement et des Ressources en Informatique Scientifique (IDRIS) of the CNRS for generous allocations of NEC SX-5 computer time. Julien Bonin and Mehran Mostafavi are gratefully acknowledged for fruitful discussions.

\section{Appendix A}

Parameters of the Pseudopotentials. From the notations in eq 10, the parameters of the original pseudopotentials are given in Table 6. The parameters obtained after optimization of the pseudopotentials (see section 2.4) are given in Table 7.

\section{Appendix B}

Gaussian Basis Sets Used in the ab Initio Calculations. We use a standard 6-311G basis ${ }^{32}$ for $\mathrm{H}_{2} \mathrm{O}$, supplemented with two d Gaussians on $\mathrm{O}$ (exponents 1.9 and 0.5 ) and two $\mathrm{p}$ Gaussians on $\mathrm{H}$ (exponents 0.8 and 0.1 ) and for the cavity electron three s and three $\mathrm{p}$ Gaussians (exponents 0.3, 0.1, and $0.03)$, one $d(0.026)$, and one $f(0.048)$ centered on the barycenter of the electron distribution. The exponents of the s and p cavity Gaussians are restricted to relatively large values: this is essential for preventing the orbitals from spreading out of the cavity. All the polarization orbitals have been optimized for the ground state at the MP2 level. For the $\left[\mathrm{e}^{-}, \mathrm{Na}^{+},\left(\mathrm{H}_{2} \mathrm{O}\right)_{28}\right]$ system we use the standard $6-31 \mathrm{G}^{*}$ basis ${ }^{32}$ for $\mathrm{H}_{2} \mathrm{O}$ and for $\mathrm{Na}^{+}$the Veillard basis. ${ }^{39}$ The use of more diffuse Gaussians in the TDSCF calculation yields an additional low-lying $\mathrm{p}$ state of the sodium atom due to finite size effect. The dipole moment of $\mathrm{H}_{2} \mathrm{O}$ using the present basis is $2.16 \mathrm{D}$, which is close to the SPC value of $2.27 \mathrm{D}$.

\section{References and Notes}

(1) Buxton, G. V.; Greenstock, C. L.; Helman, W. P.; Ross, A. B. J. Phys. Chem. Ref. Data 1988, 17, 513.

(2) Buxton, G. V.; Mulazzani, Q. G.; Ross, A. B. J. Phys. Chem. Ref. Data 1995, 24, 1055.

(3) Mostafavi, M.; Lin, M.; Wu, G.; Katsumura, Y.; Muroya, Y. J. Phys. Chem. A 2002, 106, 3123. 267.

(4) Assel, M.; Laenen, R.; Laubereau, A. Chem. Phys. Lett. 1998, 289,

(5) Asaad, A. N.; Chandrasekhar, N.; Nashed, A. W.; Krebs, P. J. Phys. Chem. A 1999, 103, 6339.

(6) Sauer, M. C.; Shkrob, I. A.; Lian, R.; Crowell, R. A.; Bartels, D M.; Chen, X.; Suffern, D.; Bradforth, S. E. J. Phys. Chem. A 2004, 108, 10414.

(7) Bonin, J.; Lampre, I.; Soroushian, B.; Mostafavi, M. J. Phys. Chem. A 2004, 108, 6817.

(8) Bonin, J.; Lampre, I.; Mostafavi, M. Radiat. Phys. Chem. 2005

(9) Gelabert, H.; Gauduel, Y. J. Phys. Chem. 1996, 100, 13993.

(10) Renou, F.; Mostafavi, M.; Archirel, P.; Bonazzola, L.; Pernot, P. J. Phys. Chem. A 2003, 107, 1506.

(11) Schlick, S.; Narayana, P. A.; Kevan, L. J. Chem. Phys. 1976, 64, 3153.

(12) Dikanov, S. A.; Tsvetkov, Y. D. J. Struct. Chem. 1985, 26, 766.

(13) Dikanov, S. A.; Tsvetkov, Y. D. Electron Spin-Echo Envelope Modulation (ESEEM) Spectroscopy; CRC Press: Boca Raton, FL, 1992.

(14) Tauber, M. J.; Mathies, R. A. J. Am. Chem. Soc. 2003, 125, 1394.

(15) Mizuno, M.; Tahara, T. J. Phys. Chem. A 2003, 107, 2411.

(16) Nicolas, C.; Boutin, A.; Lévy, B.; Borgis, D. J. Chem. Phys. 2003, $118,9689$.

(17) Spezia, R.; Nicolas, C.; Boutin, A.; Vuilleumier, R. Phys. Rev. Lett. 2003, 91, 208304

(18) Spezia, R.; Nicolas, C.; Archirel, P.; Boutin, A. J. Chem. Phys. 2004, 120, 5261 .

(19) Spezia, R.; Nicolas, C.; Coudert, F.-X.; Archirel, P.; Vuilleumier, R.; Boutin, A. Mol. Simulat. 2004, 30, 749.

(20) Spezia, R.; Coudert, F.-X.; Boutin, A. Mod. Phys. Lett. B 2004, $18,1327$.

(21) Hellmann, J. Einführung in die Quantenchemie; Deuticke: Leipzig, 1937.

(22) Feynman, R. P. Phys. Rev. 1939, 56, 340.

(23) Ewald, P. P. Ann. Phys. 1921, 64, 253.

(24) Nosé, S. J. Chem. Phys. 1984, 81, 511.

(25) Hoover, W. G. Phys. Rev. A 1985, 31, 1695.

(26) Borgis, D.; Staib, A. J. Chem. Phys. 1996, 104, 4776.

(27) Berendsen, H. J. C.; Postma, J. P. M.; van Gunsteren, W. F.; Hermans, J. In Interaction models for water in relation to protein hydratation; Reidel: Dordrecht, 1981.

(28) Aqvist, J. J. Phys. Chem. 1990, 94, 8021

(29) Turi, L.; Borgis, D. J. Chem. Phys. 2002, 117, 6186.

(30) Durand, P.; Barthelat, J. C. Theor. Chim. Acta 1975, 38, 283.

(31) Furche, F.; Ahlrichs, R. J. Chem. Phys. 2002, 117, 7433.

(32) Frisch, M. J.; Trucks, G. W.; Schlegel, H. B.; Scuseria, G. E.; Robb, M. A.; Cheeseman, J. R.; Montgomery, J. A., Jr.; Vreven, T.; Kudin, K. N.; Burant, J. C.; Millam, J. M.; Iyengar, S. S.; Tomasi, J.; Barone, V.; Mennucci, B.; Cossi, M.; Scalmani, G.; Rega, N.; Petersson, G. A.; Nakatsuji, H.; Hada, M.; Ehara, M.; Toyota, K.; Fukuda, R.; Hasegawa, J.; Ishida, M.; Nakajima, T.; Honda, Y.; Kitao, O.; Nakai, H.; Klene, M.; Li, X.; Knox, J. E.; Hratchian, H. P.; Cross, J. B.; Bakken, V.; Adamo, C.; Jaramillo, J.; Gomperts, R.; Stratmann, R. E.; Yazyev, O.; Austin, A. J.; 
Cammi, R.; Pomelli, C.; Ochterski, J. W.; Ayala, P. Y.; Morokuma, K.; Voth, G. A.; Salvador, P.; Dannenberg, J. J.; Zakrzewski, V. G.; Dapprich, S.; Daniels, A. D.; Strain, M. C.; Farkas, O.; Malick, D. K.; Rabuck, A. D.; Raghavachari, K.; Foresman, J. B.; Ortiz, J. V.; Cui, Q.; Baboul, A. G.; Clifford, S.; Cioslowski, J.; Stefanov, B. B.; Liu, G.; Liashenko, A.; Piskorz, P.; Komaromi, I.; Martin, R. L.; Fox, D. J.; Keith, T.; Al-Laham, M. A.; Peng, C. Y.; Nanayakkara, A.; Challacombe, M.; Gill, P. M. W.; Johnson, B.; Chen, W.; Wong, M. W.; Gonzalez, C.; Pople, J. A. Gaussian 03, Revision C.02; Gaussian, Inc.: Wallingford, CT, 2004.

(33) Nakano, H. J. Chem. Phys. 1993, 99, 7983.

(34) Schmidt, M. W.; Baldridge, K. K.; Boatz, J. A.; Elbert, S. T.; Gordon, M. S.; Jensen, J. H.; Koseki, S.; Matsunaga, N.; Nguyen, K. A.;
Su, S.; Windus, T. L.; Dupuis, M.; Montgomery, J. A. J. Comput. Chem. 1993, 14, 1347.

(35) Feng, D.-F.; Kevan, L. Chem. Rev. 1980, 80, 1.

(36) Kevan, L. Radiat. Phys. Chem. 1981, 17, 413.

(37) Renou, F.; Archirel, P.; Pernot, P.; Lévy, B.; Mostafavi, M. J. Phys. Chem. A 2004, 108, 987.

(38) Lide, D. R. Handbook of Chemistry and Physics; CRC Press: Boca Raton, FL, 2001.

(39) Veillard, A. Theor. Chim. Acta 1968, 12, 405.

(40) Moore, C. E. Natl. Bur. Stand. Circ. (U.S.) 1949, 467.

(41) Anbar, M.; Hart, E. J. J. Phys. Chem. 1965, 69, 1244. 